\title{
Order of the phase transition in models of DNA thermal denaturation
}

\author{
Nikos Theodorakopoulos ${ }^{1,2}$, Thierry Dauxois ${ }^{2}$ and Michel Peyrard ${ }^{2}$ \\ 1 Theoretical and Physical Chemistry Institute, National Hellenic Research Foundation \\ Vasileos Constantinou 48, 11635 Athens, Greece \\ ${ }^{2}$ Laboratoire de Physique, UMR-CNRS 5672, ENS Lyon, 46 Allée d'Italie, 69364 Lyon Cédex 07, France
}

(submitted to Physical Review Letters on January 5, 2000; revised March 31, 2000)

We examine the behavior of a model which describes the melting of double-stranded DNA chains. The model, with displacement-dependent stiffness constants and a Morse onsite potential, is analyzed numerically; depending on the stiffness parameter, it is shown to to have either (i) a second order transition with $\nu_{\perp}=-\beta=1, \nu_{\|}=\gamma / 2=2$ (characteristic of short-range attractive part of the Morse potential) or, (ii) a first-order transition with finite melting entropy, discontinuous fraction of bound pairs, divergent correlation lengths, and critical exponents $\nu_{\perp}=-\beta=1 / 2, \nu_{\|}=\gamma / 2=1$.

PACS numbers: 05.70.Jk, 63.70.+h, 87.10+e, 05.70.Fh

Early models of DNA thermal denaturation, i.e. the separation of the two strands upon heating [1], were based on an Ising-like description in which a base-pair was either closed or open; the relative tendencies of the system to occupy one of the two states were introduced explicitly, in terms of temperature-dependent free enthalpies. As a consequence, although a judicious choice of such enthalpies has proved useful in describing some aspects of DNA denaturation [2], understanding of this remarkable one-dimensional cooperative phenomenon in terms of standard statistical mechanics -i.e. a Hamiltonian model with temperature-independent parametersremained an outstanding problem.

More recent research has emphasized the role of the large amplitude fluctuations that precede the transition and the intrinsically nonlinear mechanisms which are needed to describe such fluctuations [3]. In such models the status of a base pair is characterized by the distance between the two bases. An on-site asymmetric potential with a flat part at large values of the displacement emulates the tendency of the pair to "melt" at high temperatures, as thermal phonons drive the particles outside the well and towards the flat portion of the potential.

In the original version ("type I" model) coupling between successive base pairs is harmonic [4]; the resulting path to the melting instability appears smooth; this is at variance with the sharp features of the transition observed experimentally. A generalization [5,6] of the model to include displacement-dependent stiffness constants, i.e. "stacking parameters" which describe the coupling between successive base pairs, has revealed a dramatic sharpening of the transition. The predictions of the latter, ("type II") model have been compared successfully with experimental results [7]. Furthermore, investigations of heterogeneous DNA have shown that the model yields features of multistep melting similar to those observed in experiments [8]. In fact, the authors of Ref. [8] have, in passing, pointed out the formal analogies between the melting instability of homogeneous DNA and other continuous phase transitions, e.g. wetting of a one-dimensional interface from a substrate, adsorption of polymers by a solid surface etc.; in addition, they have demonstrated that "type II" models generate an "entropic barrier" which is largely responsible for the narrowing of the transition. Their analysis of the order parameter led them however to suggest that, in spite of the dramatic narrowing of the transition, the critical exponent remains unchanged. As a result, the exact character of the homogeneous DNA transition remained somewhat elusive.

In this Letter we report on the scaling behavior of typeII model of the denaturation of ideal, homogeneous DNA. We show that, for values of the stacking parameter used in [6] the type-II model exhibits a peculiar type of first order transition, with a finite melting entropy, a discontinuity in the fraction of bound pairs (the usual DNA observable), and divergent longitudinal and transverse correlation lengths [9]; as the value of the stacking parameter changes, and the range of the "entropic barrier" becomes shorter than, or comparable to the range of the Morse potential, the transition changes to second order, as in type-I models.

The Hamiltonian of the model is

$$
H=\sum_{n}\left[\frac{p_{n}^{2}}{2 m}+W\left(y_{n}, y_{n-1}\right)+V\left(y_{n}\right)+\text { Dhay }_{n}\right]
$$

where $m$ is the reduced mass of a base pair, $y_{n}$ denotes the stretching of the hydrogen bonds connecting the two bases of the $\mathrm{n}^{\text {th }}$ pair and $p_{n}=m\left(d y_{n} / d t\right)$. Coupling between successive base pairs is described by $W\left(y_{n}, y_{n-1}\right)=\frac{K}{2}\left[1+\rho e^{-\alpha\left(y_{n}+y_{n-1}\right)}\right]\left(y_{n}-y_{n-1}\right)^{2}$. The parameter $\rho$ can take non-zero values in type-II models; The choice of this coupling potential is motivated by the observation that the stacking energy is a property of base pairs rather than individual bases. The effective coupling constant decreases from $(1+\rho) K$ to $K$ when either one of the two interacting base pairs is open, in qualitative agreement with the known properties of base-pair interactions in DNA. The third term in (11) stands for an on-site potential which describes the 
interaction of the two bases in a pair; the Morse potential $V\left(y_{n}\right)=D\left(e^{-a y_{n}}-1\right)^{2}$ has been chosen because it has the correct qualitative shape. Finally, the fourth term, which describes the effect of a transverse, external stress $h$, is in fact a mathematical device useful in practical calculations. By letting the dimensionless $h$ approach zero from above, it is possible to extract the scaling behavior near the transition; at the same time, since the partition function is now divergence-free at any $h>0$, a source of criticism of the model on formal mathematical grounds [10] is removed. The parameters of the model are: $D=0.03 \mathrm{eV}, K=0.06 \mathrm{eV} / \stackrel{\circ}{A}^{2}, a=4.5 \stackrel{\circ}{A}^{-1}$, $\alpha=0.35 \AA^{-1}, m=300$ a.m.u. and the lattice constant $l=4.5 \stackrel{\circ}{A}$.

The thermodynamic properties of (11) can be described [11] in terms of the eigenvalues and eigenstates of the transfer-integral (TI) equation

$$
a \int_{-\infty}^{\infty} d y e^{-\mathcal{K}(x, y) / k_{B} T} \phi_{i}(y)=e^{-\varepsilon_{i} / k_{B} T} \phi_{i}(x)
$$

with a symmetrized kernel $\mathcal{K}(x, y)=W(x, y)+\frac{1}{2}[V(y)+$ $V(x)]+\frac{D a h}{2}(y+x)$; here $T$ is the temperature and $k_{B}$ the Boltzmann constant. Details of the numerical procedure used in solving (2) have been given in [6]; in the present study a Gauss-Legendre quadrature formula has been used. In the gradient expansion (continuum) approximation, valid in the temperature window $D<k_{B} T<K / a^{2}$ [12], the integral equation (2) can be well approximated by the second-order differential equation

$$
-\frac{1}{\delta g(x) a^{2}} \frac{d^{2} \phi_{i}}{d x^{2}}+U(x) \phi_{i}=\tilde{\epsilon}_{i} \phi_{i}
$$

where $\delta \equiv \sqrt{2 K D / a^{2}} /\left(k_{B} T\right), g(x)=1+\rho \exp (-2 \alpha x)$, $D \tilde{\epsilon}_{1}=\epsilon_{i}+\left(k_{B} T / 2\right) \ln \left(2 \pi a^{2} k_{B} T / K\right), D U(x)=V(x)+$ $\left(k_{B} T / 2\right) \ln g(x)$, and the limit $h \rightarrow 0$ has been explicitly taken.

Of particular interest are (i) the lowest eigenvalue $\epsilon_{0}$, which, in the thermodynamic limit, is equal to the free energy per site $f$, (ii) the ground state $\phi_{0}$, which determines the order parameter $\sigma \equiv\langle y\rangle \equiv \int d y y\left|\phi_{0}\right|^{2}$ and its fluctuations $\left\langle(\delta y)^{2}\right\rangle \equiv \int d y(y-\sigma)^{2}\left|\phi_{0}\right|^{2}\left(\equiv \xi_{\perp}^{2}\right.$, the transverse correlation length in the language of wetting [13]), and (iii) the next-to-lowest eigenvalue, which controls the longitudinal correlation length $\xi_{\|}=l k_{B} T /\left(\epsilon_{1}-\epsilon_{0}\right)$. Computation of the static structure factor $S(q, T)=$ $\sum_{n} \exp (-i q n l)\left\langle\delta y_{n} \delta y_{0}\right\rangle$, where $\delta y_{n}=y_{n}-\sigma$, requires knowledge of the full spectrum; in terms of the matrix elements $M_{i} \equiv\langle i|x| 0\rangle$, and the differences $\Delta_{i} \equiv$ $\left(\epsilon_{i}-\epsilon_{0}\right) / k_{B} T$,

$$
S(q, T)=\sum_{i}{ }^{\prime}\left|M_{i}\right|^{2} \frac{\sinh \Delta_{i}}{\cosh \Delta_{i}-\cos (q l)},
$$

where the ground state is excluded from the summation.
For $\rho=0$ (type - I case) the asymptotic properties of the denaturation instability can be obtained from the solution of the "pseudoSchrödinger" Eq.(3) with $g=1$ 114,15. In brief: as long as $1>\delta>\delta_{c}=1 / 2$, there is a single bound state with $\tilde{\epsilon}_{0} / D=1-\left(1-\delta_{c} / \delta\right)^{2}$ which disappears at $\delta=\delta_{c}$, corresponding to a critical temperature $k_{B} T_{c}=2 \sqrt{2 K D} / a$; in the critical regime preceding the instability, $|t| \ll 1, t \equiv T / T_{c}-1$, the power laws $l / \xi_{\|} \propto|t|^{\nu_{\|}}, \sigma \propto|t|^{\beta}$ and $\xi_{\perp} \propto|t|^{-\nu_{\perp}}$ hold, with $\nu_{\|}=2$ and $\nu_{\perp}=-\beta=1$. Furthermore, we have calculated the structure factor 16 in the regime $q a \ll 1, \xi_{\|} / l \gg 1$,

$$
S(q, T)=\frac{1}{a^{2}\left(\delta-\delta_{c}\right)^{2}} \frac{\xi_{\|}}{l} F\left(q \xi_{\|}\right),
$$

where $F(x)=(1 / 4) x^{-2}[1-1 / \cosh (\operatorname{arcsinh}(x) / 2)]$. This implies (i) a zero-field isothermal susceptibility $\chi=$ $\lim _{h \rightarrow 0} a(\partial \sigma / \partial h)_{T} \propto|t|^{-\gamma}$, where $\gamma=4$, and (ii) critical correlations $\left(q \xi_{\|} \gg 1\right), S(q) \propto(q l a)^{-2+\eta}$ with $\eta=0$.

It should be noted that the occurrence of a thermodynamic transition in the one-dimensional model (11) does not imply a violation of well-known theorems: vanHove's theorem [17 does not apply, since the Hamiltonian includes an on-site term; furthermore, since there is no symmetry-breaking (or domain-wall-like solitons) involved, the standard Landau argument against phase transitions in one dimension is also inapplicable.

Numerical results for a type-II model $(\rho=1, \alpha=$ $\left.0.35 \AA^{-} 1, \alpha / a=0.078\right)$, obtained by solving the TI equation (2) for various values of $T$ and $h$, are summarized in Fig. 1 [18. Below $T_{c}$, the difference between the two lowest eigenvalues, which determines $\xi_{\|}$as well as the singular part of the free energy, is found empirically to satisfy the scaling equation

$$
\epsilon_{1}-\epsilon_{0}=-f_{\text {sing }}=D|t| \Phi\left(\frac{h}{|t|^{3 / 2}}\right),
$$

with the limiting forms $\Phi(x)=\Phi_{0}+\Phi_{1} x+\Phi_{2} x^{2}+\ldots(x \ll$ 1) and $\Phi(x) \propto x^{2 / 3}(x \gg 1)$; it follows that $\nu_{\|}=1, \gamma=2$ and $-\beta=1 / 2$. The values of the first two critical exponents are in very good agreement with those obtained directly from our zero-field results for the longitudinal correlation length and the susceptibility, respectively(cf. Fig. 2). The order parameter and, to a lesser extent, the transverse correlation length (also shown in Fig. 2) reveal significant deviations from pure power-law behavior - presumably due to strong transients (cf below); results are however roughly consistent with $\nu_{\perp}=1 / 2$.

It is possible to follow the transition from type-I to type-II behavior by continuously varying the stacking parameter $\alpha$, at constant $\rho=1$. We have done this using numerically evaluated semiclassical (Bohr-Sommerfeld) eigenvalues of Eq. 3. The results, shown in Fig. 3, indicate that a crossover takes place at $\alpha / a \approx 0.5$. Smaller values of $\alpha / a$ correspond to a longer -yet finite- range of the entropic barrier, compared with that of the Morse 
potential; one obtains the type-II exponent, $\nu_{\|}=1$. As $\alpha / a$ increases and the range of the entropic barrier becomes shorter than that of the Morse potential, the entropic barrier becomes irrelevant to critical behavior; one obtains the type-I exponent, $\nu_{\|}=2$.

Equation (6) states that the singular part of the zerofield free energy depends linearly on the temperature. In other words, the value of the exponent $\nu_{\|}=1$ implies a first-order transition. For our parameter set, the corresponding melting entropy is $\Delta S / k_{B}=\left[D /\left(k_{B} T_{c}\right)\right] \Phi_{0}=$ .75 (cf inset in Fig. 4).

At this point a comment on the fraction of bound pairs is in order; it is this quantity which one measures for DNA, using UV absorbance, rather than the order parameter. In the type-I model, the probability of finding a given base pair at an equilibrium distance smaller than $b$ (equal to the fraction of bound pairs, with a proper choice of $b)$, is given in terms of the incomplete gamma function, i.e. $P(y<b, T)=1-\gamma\left(2 \delta-1,2 \delta e^{-a b}\right) / \Gamma(2 \delta-1)$, and approaches zero continuously as $T \rightarrow T_{c}$, independently of the choice of $b$. This is not the case in type II behavior. It can be seen in Fig. 4 that there is a step discontinuity in the fraction of bound pairs; the exact magnitude of the step depends on the choice of $b$, but the discontinuity appears to be an intrinsic property of the type-II ground state wave function; the subtlety lies in the fact that, although there is a long-tail which causes the divergence of $\sigma$, a finite weight of $\left|\phi_{0}\right|^{2}$ originates in finite displacements. This is to be contrasted with type-I behavior, where more and more weight is shifted to infinity as the transition is approached. Therefore, the experimental detection of the fraction of bound pairs in terms of the function $P(y<b, T)$, provides accurate information about the true order of the transition; the order parameter itself diverges smoothly at the transition and might, due to transients which mask the leading-order asymptotics, be less suited for a detailed study, even if it were readily available by experimental methods. This is a fortunate natural coincidence.

In summary, we have presented a complete scaling analysis of a simple model which has been developed in order to describe the melting of "homogeneous" DNA. In terms of biophysical applications, our results should be complemented by the analysis of Ref. [8] to account for the effects of heterogeneity. We feel nonetheless, that there is a direct gain from the analysis presented here: the melting of a double-stranded chain - a fairly general problem of biologically motivated statistical physics - has been shown to be a true thermodynamic transition with different types of critical behavior, depending on the details of the interaction parameters. It would be interesting to speculate on whether other varieties of thermal biomolecular denaturation (e.g. protein unfolding) could be studied in terms of related models of low-dimensional phase transitions.

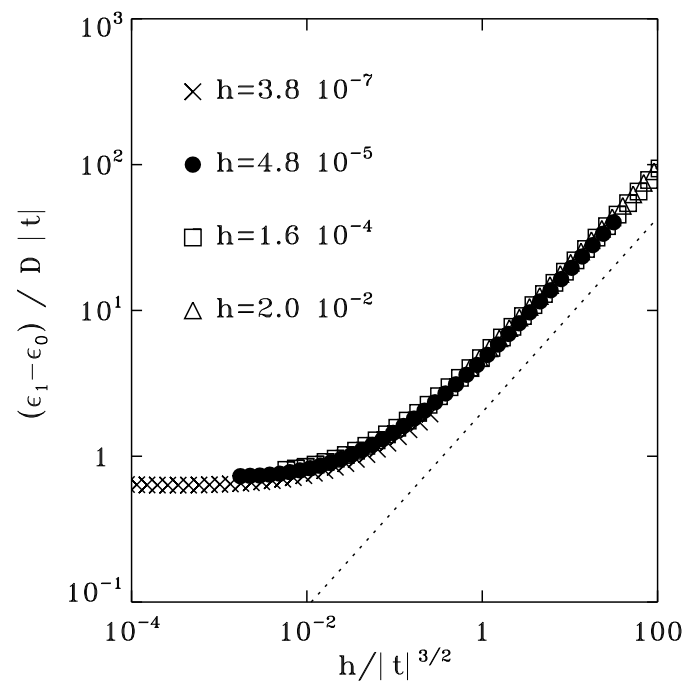

FIG. 1. Type II critical behavior: the dependence of $\left(\epsilon_{1}-\epsilon_{0}\right) / D|t|$ on the scaling variable $h /|t|^{3 / 2}$ is shown for 4 different values of $h$ and a range of temperatures; the dotted line marks the slope $2 / 3$.

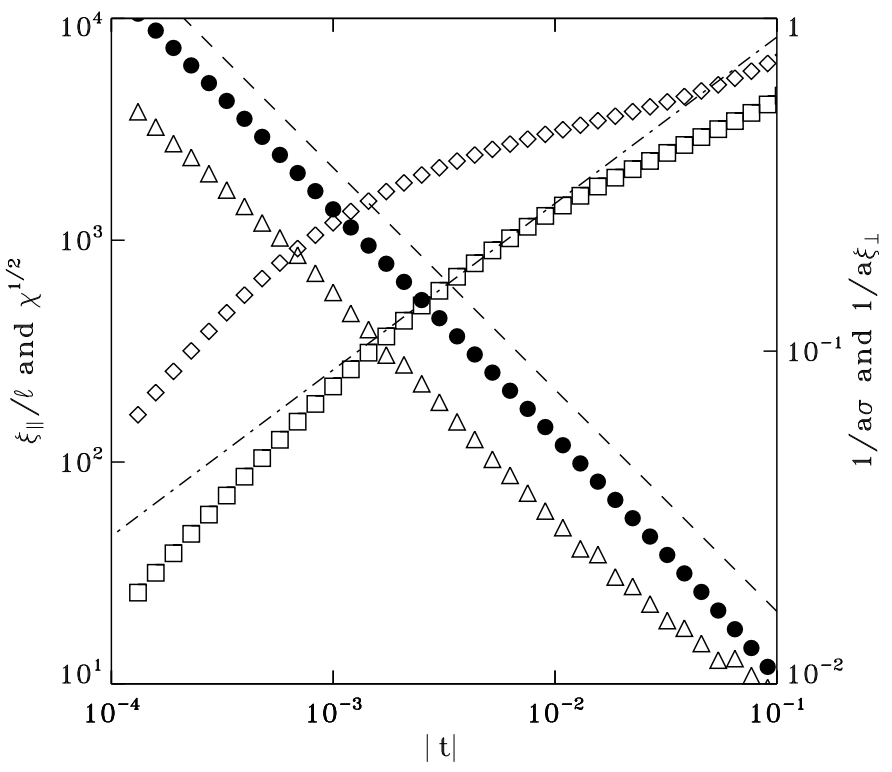

FIG. 2. Zero field results in the type-II case. The longitudinal correlation length $\xi_{\|}$(circles, left axis), the square root of the susceptibility $\chi$ (triangles, left axis), the order parameter $\sigma$ (diamonds, right axis) and its root-mean-square fluctuations $\xi_{\perp}$ (squares, right axis) as a function of $|t|$. The dashed and dashed-dotted lines correspond to $\nu_{\|}=\gamma / 2=1$ and $\nu_{\perp}=1 / 2$, respectively. 


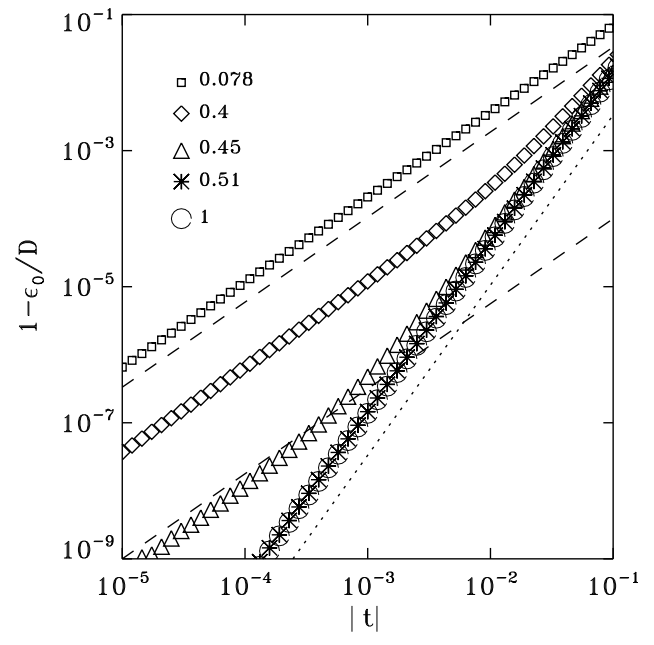

FIG. 3. The difference between the lowest eigenvalue of Eq. 3 and the bottom of continuum band, as a function of the reduced temperature, calculated numerically for various values of the ratio $\alpha / a$, within the Bohr-Sommerfeld quantization scheme. The asymptotic slope, which gives the critical exponent $\nu_{\|}$, changes from 1 to 2 , as the stacking parameter varies from type-II $(\alpha / a<1 / 2)$ to type-I $(\alpha / a>1 / 2)$ values. The dashed and dotted lines have slopes of 1 and 2 , respectively.



FIG. 4. The fraction of bound base pairs $P(y<2 \AA, T)$ as a function of the $T / T_{c}$ for the type I (triangles) and II (diamonds). Inset: the entropy $S(T) / N k_{B}$ ) (symbols as in main fig.); the length of the double arrow represents the estimate of the melting entropy obtained from the scaling Eq. (6). The solid lines are "guides to the eye".
[1] R.M. Wartell and A.S. Benight, Physics Reports 126, 67 (1985).

[2] D. Poland and H. A. Scheraga, J. Chem. Phys. 45, 1456 (1966), ibid 45, 1464 (1966).

[3] S.W. Englander, N.R. Kallenbach, A.J. Heeger, J.A. Krumhansl, and S. Litwin, Proc. Nat. Acad. Sci. (USA), 777, 7222 (1980)

[4] M. Peyrard and A.R. Bishop, Phys. Rev. Lett. 62, 2755 (1989).

[5] T. Dauxois, M. Peyrard and A. R. Bishop, Phys. Rev. E 47, R44 (1993).

[6] T. Dauxois and M. Peyrard, Phys. Rev. E 51, 4027 (1995)

[7] A. Giansanti and A. Campa, Phys. Rev. E 58, 3585 (1998).

[8] D. Cule and T. Hwa, Phys. Rev. Lett. 79, 2375 (1997).

[9] First-order transitions with divergent correlation lengths have been previously reported in the context of wetting: V. Privman and N. M. Svrakic, Phys. Rev. B 37, 5974 (1988); R. Blossey and J. O. Indekeu, Phys. Rev. E 52, 1223 (1994).

[10] Y-L Zhang, W-M Zheng, J-X Liu, Y. Z. Chen, Physical Review E 56, 7100 (1997).

[11] J. A. Krumhansl and J. R. Schrieffer, Phys. Rev. B 11, 3535 (1975).

[12] R. A. Guyer and M. D. Miller, Phys. Rev. A 17, 1205 (1978).

[13] D. M. Kroll and R. Lipowski, Phys. Rev. B 28, 5273 (1983); R. Lipowski, Phys. Rev. B 32, 1731 (1985).

[14] L. D. Landau and E. M. Lifshitz, Quantum Mechanics, non-relativistic theory, page 72 (3d Edition, Pergamon, 1977); M. M. Nieto and L. M. Simmons, Phys. Rev. A 19, 438 (1979).

[15] There is an extensive mathematical literature on weakly bound eigenstates of the one-dimensional Schrödinger equation. We mention here the work of (a) B. Simon, Ann. Phys. (NY) 97, 279 (1976), (b) M. Klaus, ibid 108, 288 (1977), (c) M. Klaus and B. Simon, ibid 130, 251 (1980), and (d) R. Blanckenberg, M.L. Goldberger and B. Simon, ibid 108, 69 (1977). The problem with applying the conclusions of these papers to the present problem is that they impose various requirements of boundedness on the potential, which the repulsive core of the Morse potential cannot meet. Interestingly, paper (c) predicts in the case of three dimensions and for states of nonzero angular momentum (i.e. with a rotational barrier), linear, rather than quadratic dependence of the bound state energy on the distance from threshold; this does parallel our findings on the critical exponent $\nu_{\|}$for cases with and without entropic barrier, respectively.

[16] The details of the calculation will be reported elsewhere.

[17] L. van Hove, Physica 16, 137 (1950).

[18] The transition temperature was estimated by exploiting the "topological" difference occurring in a family of $\sigma(T, h)$ vs. $T$ plots for various small fields: all the "low temperature" plots give a finite limit as $h \rightarrow 0$; the "high temperature" plots give a divergent $\sigma(T, 0)$. The procedure determines $T_{c}$ within a relative error of $3 \times 10^{-5}$. 